\title{
ANALYSIS OF COMPLEXITY AND SIMULATION USAGE IN MANUFACTURING SMES
}

\section{Dr. James Byrne}

DCU Business School, Dublin City University, Glasnevin, Dublin 9, Ireland james.byrne@dcu.ie

\section{Dr. Paul Liston}

\author{
Dept. of Accounting \& Business Computing \\ Faculty of Business and Hospitality \\ Athlone Institute of Technology, Ireland \\ pliston@ait.ie
}

\author{
Prof. PJ Byrne \\ DCU Business School, \\ Dublin City University, \\ Glasnevin, Dublin 9, Ireland \\ pj.byrne@dcu.ie
}

\begin{abstract}
Discrete event simulation (DES) is a powerful decision support methodology particularly suited to complex systems where the relationship between individual components is difficult to comprehend. This study aims to investigate DES usage and its relevance in manufacturing SMEs using a surveybased calculated complexity value for each organisation. A study was conducted to assess Irish manufacturing SMEs' perception of their organisation's complexity versus a survey-based calculated complexity value. Complexity levels were then used to determine the potential for DES usage. Respondents were also asked to respond on DES awareness and usage. This study suggests that manufacturing SMEs seem to underestimate the complexity of their systems and consequently the usefulness of DES. The poor uptake of DES among manufacturing SMEs can be partly attributed to a lack of awareness. This paper presents a call to action by the DES modelling community including practitioners, academics and software vendors in support of manufacturing SMEs.
\end{abstract}

Keywords: Discrete Event Simulation, DES, Complexity, Small and Medium Enterprises, SME

\section{INTRODUCTION}

Manufacturing small and medium-sized enterprises (SMEs) face a number of operational challenges such as scheduling, flexibility and lead time, customization, product returns, maintenance operations and the supply chain to note just a few. What is notable is that these challenges are not specific to companies of SME status but are also regularly encountered by larger organizations. However, the unique characteristics of manufacturing SMEs affect the conditions required to work with manufacturing strategies (Löfving et al., 2014). In addition, manufacturing SMEs are consistently challenged to improve efficiencies as they compete in a global market against large well-resourced multinational companies. In efforts to address such operational (and also strategic) challenges, larger organizations quite often evaluate the manufacturing technology landscape and the potential solution supports this makes available to them. Discrete Event Simulation (DES) is one such tool which can be applied in such situations. While some larger companies have gained from this methodology, smaller firms seem less likely to take advantage of its capabilities (Ingemansson et al., 2002). This low uptake by SMEs has occurred despite DES being available for a considerable period of time and its benefits being well publicized. Given the independent and peer-reviewed validation of the advantages of the use DES including dedicated academic journals, conferences and workshops on the subject there are a 


\section{Byrne, Liston, and Byrne}

number of open and as of yet unanswered questions in this regard: why is DES modelling not ubiquitous across the entire manufacturing sector? Do companies think that DES is not suited to their specific problem types? Is there an inherent aversion to this form of analysis? How many of those at the "coalface" are actually aware of DES? It is generally accepted that DES, although beneficial, can be costly and time consuming to undertake (Fowler and Rose, 2004; Johansson et al., 2008). It is proposed that the context in which DES offers greatest return is one where the system under investigation is sufficiently complex to justify analysis beyond standard spreadsheet-based calculations. Indeed, Robinson (2005) notes that "simulations are normally developed because a system is too complex to be represented in any other way". The challenge with this perspective is that 'complexity' is, in and of itself, difficult to define. There has been extensive research on the subject of complexity with many publications offering insight from different perspectives including that of manufacturing (e.g. Garzon et al. (2012); Lang et al. (2014)), and the topics of simulation and complexity have been considered together e.g. Zhang (2011); Aelker et al. (2013).

In this work, the authors aim to examine the level of complexity across a sample of companies in the Irish manufacturing sector and indicate how this can affect their potential use of DES. To this end, a brief overview of the literature on SME technology strategy and the broader topic of complexity is presented, followed by details of the data collection and analysis methodologies, the consequent findings and finally the conclusions drawn from the research.

\section{LITERATURE REVIEW}

The concept of complexity has been studied in great detail and the literature is comprehensive and varied in relation to the approaches and perspectives taken. Studies of complexity have been conducted across a large array of disciplines and areas in the manufacturing domain such as understanding the architecture of complexity (Simon, 1962); investigating the complexity of scheduling in SMEs (Garzon et al., 2012); determining the impact of manufacturing complexity on performance (Pradhan and Damodaran, 2009) and managing complexity (Götzfried, 2013). The meaning of the word complexity is considered to be vague and ambiguous (ElMaraghy et al. 2012) with others affirming that there is no real universally accepted definition or consistent interpretation of complexity (Aelker et al., 2013). In their attempts to understand complexity many have sought to determine how it can be quantified or measured (e.g. Modrak and Marton 2012) while others have focused on how it can be modelled and simulated (e.g. Cicirelli et al., 2011; Zhang, 2011). Some systems can be so complex as to be almost impossible to fully understand. Indeed Beer (1959) argued that in the world there exists a class of "exceedingly complex systems", which are in principle unknowable, we can never know them completely and they can always surprise us, including the brain, firm and economy (Pickering, 2004). Much work has been done in the area of complex adaptive systems (CAS) theory (e.g. see Anderson (1999), Lewin (1999), Choi et al. (2001)) in which a perfect understanding of the individual parts of a system does not automatically convey a perfect understanding of the whole system's behavior (Miller and Page, 2017). In all, a significant volume of complexity-related literature has been published. In determining the nature of complexity studies four categories have been identified in relation to the different types of complexity that can exist. The predominant categories of complexity that have emerged are static and dynamic complexity (Gabriel, 2008; Serdarasan, 2013) and internal and external complexity (Gabriel, 2008; Götzfried, 2013).

- Static complexity can be referred to as structural complexity (ElMaraghy et al., 2012), which is time-independent and intrinsic in the product and systems structure (Gabriel, 2008).

- Dynamic complexity relates to the unpredictability in the behaviour of a system over time and is thus time dependent and related to the operational behaviour of the company (ElMaraghy et al., 2012). Gabriel (2008) notes that the events in manufacturing systems that lead to dynamic complexity include machine breakdowns and quality failures.

- Internal complexity is related to the complexities experienced within the company and is considered endogenous (Götzfried, 2013). The facets which are deemed to be in control of the managers, such as product offerings, types and amount of equipment, degree of vertical integration, systems design and maintenance, reliability, quantity and timing of materials and tools all contribute to internal complexity (Gabriel, 2008). 


\section{Byrne, Liston, and Byrne}

- External complexity relates to those factors which are outside the control of the company and its managers, and include factors such as: customer expectations and demands; regulations and market and environmental changes; intensifying competition; external stakeholders such as customers, suppliers, distributors and regulatory bodies (Blome et al., 2014; Efthymiou et al., 2012; Gabriel, 2008; Götzfried, 2013).

Many features of manufacturing environments contribute to complexity and several frameworks for outlining the drivers of complexity have been devised (Aelker et al., 2013). Following a literature analysis this paper proposes six general complexity categorizations (determinants), see Table 1.

\section{Table 1 Determinants of Complexity}

\begin{tabular}{|l|l|}
\hline Product/Parts & $\begin{array}{l}\text { High product mix; Multiple part types made in the same facility/line; Multiple } \\
\text { levels of subassemblies; Product features and capabilities; Electrical and } \\
\text { mechanical components, software, and human-interfaces; }\end{array}$ \\
\hline Process & $\begin{array}{l}\text { Number of process technologies; Number of manufacturing steps; Batch } \\
\text { processing; Automation: highly integrated with all levels of the enterprise; Layout } \\
\text { of components in a system and connectivity between them; Number of routes } \\
\text { through the factory; Information flow: internal, external and intra-plant; Planning } \\
\text { and scheduling functions: strategies, number, content, timing \& priority of } \\
\text { documents for planning and scheduling, decision making; Precedence constraints } \\
\text { for set of operations for producing a part type; Relationships between } \\
\text { parts/components; Many elements with numerous but simple interrelations; }\end{array}$ \\
\hline Equipment & $\begin{array}{l}\text { Complex equipment that leads to high levels of preventive maintenance and } \\
\text { downtime; Multiple products made on different machines; Control systems and } \\
\text { software; Machine capabilities; Unpredictability over time; }\end{array}$ \\
\hline People & \begin{tabular}{l} 
Operator absenteeism; Human cognitive ergonomics; \\
\hline Supply Chain
\end{tabular} \\
\hline Market & $\begin{array}{l}\text { Delays and faults in raw materials; Resource unavailability; } \\
\text { delivery, zero defects; Customer change requests; Shorter product lifecycles; More } \\
\text { intense competition; Rising customer expectations; Social and environmental } \\
\text { pressures; Government legislation and standards; }\end{array}$ \\
\hline
\end{tabular}

In terms of the measurement of complexity, Frizelle and Woodcock (1995) developed a mathematical model utilizing entropy as a measure of the complexity in a system. Their proposed method considers the elements of a system, the extent to which they interact and the degree to which each operational source contributes to the overall complexity of the firm. Validation of the method was presented by way of three case studies. Further validation of the entropy method was provided by Frizelle and Suhov (2008) where an additional three cases were tested. However, no evidence was found of widespread usage beyond these validation cases. Around the same time the Meyer and Foley Curley (MFC) approach was developed (Meyer and Curley, 1995). The MFC approach introduces the concepts of knowledge and technology complexity, uses a scoring methodology for defined system variables and was found to give good insight at a relatively low cost. In a study of the two approaches Calinescu et al. (1998) found that although the entropic method gave greater insight, it had a significantly higher input cost. Examples of researchers trying and failing to successfully measure complexity are also available. Gabriel (2008) proposed and tested a quantitative measure for the manufacturing complexity that results from system design, referred to as the internal static manufacturing complexity (ISMC) measurement. The ISMC was focused on product line complexity, product structure and process complexity components and consisted of eight measurable factors.

In studies associated with measuring complexity in manufacturing environments, there is often a written or unwritten assertion that complexity is a negative attribute and that the proposed measure of complexity can either be used to identify the less complex option when making decisions or as a yardstick for process improvement initiatives. Deshmukh et al. (1998) however argue that an increase in static complexity can in fact result in an improvement in system performance if the system is operated optimally. They note, that for a given part mix, static complexity can be increased by adding machines to the shop floor, changing the capability of some machines so that they can handle more 


\section{Byrne, Liston, and Byrne}

operations, and by changing the process plans for parts such that the operations can be processed in any sequence. The foregoing descriptions demonstrate only a small sample of the efforts to observe, assess and develop measures for gauging how the various components of a business environment can lead to complexity. However, despite the breadth of literature, little is published in relation to complexity in manufacturing SMEs and more specifically the role that DES for such SMEs may hold.

\section{METHODOLOGY}

This study aims to better understand why DES uptake is low, particularly amongst SMEs, and determine if there is justification for attempting to improve this metric. To gain the perspective of this business sector, the opinions and details of a representative sample of manufacturing SMEs based in Ireland were collected and studied. With the research objectives in mind, a survey was selected as the most appropriate data collection mechanism and a set of questions was compiled based on past DES study experience of the research team and the relevant literature. With no readily available dataset of potential respondents, a number of steps were undertaken prior to the survey being conducted. The first step involved defining the participant category of interest (i.e. companies that meet the SME criteria and operate in the manufacturing sector). Using these criteria, a dataset of 500 Irish manufacturing SMEs was created through contacting local and national enterprise boards and government bodies and reviewing media reports. Information was collated on company name, type, turnover (where possible) and contact details.

DES requires a certain amount of complexity in the system of interest in order for companies to realize the maximum value from the model. Without this level of complexity other tools such as spreadsheet modelling and queuing methods may provide a better solution for SMEs striving to obtain an understanding of, and evaluate proposed changes in, their processes (Chance et al., 1996; Fowler and Rose, 2004). Consequently, in addition to gaining details of the companies and their attitude toward DES, one objective of the survey was to establish a sense of the complexity within respondents' systems. As noted from the literature, defining, understanding and measuring complexity is not an exact science. Nonetheless, previous studies have built up a knowledgeable base from which to understand the concept of complexity. Bar Garzon et al. (2012) no other literature was found which focuses on the topic of SME complexity with most studies dealing with large organizations. Garzon et al. (2012) focused on scheduling complexity and does not offer a broader framework or set of measures to apply to manufacturing SME complexity. With no clearly defined set of criteria with which to measure SME complexity, reference was made in this study to the literature on the determinants of complexity (see Table 1) and the criteria used in past DES studies.

Given that companies were being studied remotely via survey, the time and effort required to engage in the in-depth complexity measurement processes (e.g. the entropy rate or MFC methods) was deemed prohibitive. Instead, a simpler two-pronged (perception versus calculation) approach was designed and implemented. The first element of this approach was a direct question asking respondents for their own perception of their own systems' complexity. With the exception of presenting system parameters that may contribute to complexity, no definition of complexity was offered which allowed respondents to determine their own perception of their own systems' complexity based on their own assumptions of the complexity they experience in their manufacturing system. Specifically, the question posed was "How complex do you think your production system is? (For example, in relation to the number of products, the range of processes, product crossovers, variability, etc.)" with the possibility of answering "very", "somewhat", or "not very complex". The second element consisted of a set of questions pertaining to the complexity determinants. The information sought (Table 2) reflected the fact that a company's complexity can be affected by both internal (e.g. batch sizes) and external factors (e.g. customer demand) and covers the main determinant categories of complexity in manufacturing as presented in Table 1. In the questions for batch size, demand and production variability, the respondents could choose from a three point scale and each point was mapped directly back to a complexity level of "very", "somewhat", or "not very complex". The two questions related to SKUs were open-ended to allow respondents to describe their own particular case and therefore categorization was required prior to mapping to a complexity level. Once the response for each question was mapped to a complexity level, it was possible to compare the average calculated complexity level with the perceived complexity level as SME self-assessment. 
Respondents were also asked questions under three other broad categories, company overview, IT system usage and DES awareness.

Table 2 Criteria requested to gauge manufacturing SME complexity

\begin{tabular}{|l|l|l|}
\hline \multicolumn{1}{|c|}{ Criteria } & \multicolumn{1}{|c|}{ Information sought from survey respondents } & \multicolumn{1}{c|}{ Determinant } \\
\hline Batch size & $\begin{array}{l}\text { Do batch sizes always remain the same, change somewhat } \\
\text { or change constantly? }\end{array}$ & Products \\
\hline Total SKUs & How many different SKUs exist in the system? & Products \\
\hline Active SKUs & How many active SKUs are live at any one time? & Products \\
\hline $\begin{array}{l}\text { Customer } \\
\text { demand }\end{array}$ & $\begin{array}{l}\text { Does customer demand always remain the same, change } \\
\text { somewhat or change constantly? }\end{array}$ & $\begin{array}{l}\text { Market/Supply } \\
\text { Chain }\end{array}$ \\
\hline Production & $\begin{array}{l}\text { Is production variability considered to be low, medium or } \\
\text { high? }\end{array}$ & $\begin{array}{l}\text { Process/Equipment/ } \\
\text { People }\end{array}$ \\
\hline
\end{tabular}

The survey was distributed to all 500 Irish manufacturing SMEs on the developed distribution list and held open for 12 weeks. Companies were mostly contacted via email or, where no direct email was available, contact was made through the company contact form on their website. A follow up strategy was set in place whereby each company received a maximum of two further emails after the initial contact was made. Perhaps due to bad destination email boxes twelve invites did not successfully reach the companies. Out of the 488 emails that were successfully delivered, responses were received from 183 of the SMEs (37.5\%). However, after initial review 111 fully completed surveys were found to be usable for the purpose of assessing complexity $(22.5 \%)$. Out of the usable surveys, it is noted that over $85 \%$ of survey respondents occupied a "Director" level role, with a further 5\% operating at "Senior Management" level, with the remaining 9.5\% of respondents operating at a level below "Director" or "Senior Management" (with described roles such as: accounts personnel, administrators, secretaries, laboratory analysts).

\section{RESULTS}

An overview of the companies surveyed is provided in Table 3, where the time in business and scale in terms of turnover and number of employees is presented. As an indication of IT adoption behavior, company usage of cloud-based data storage is also charted. It can be seen that the majority were well established companies (greater than 10 years in business) with a broad range of annual turnovers (mostly less than 5 million euro per annum). Most companies were small in terms of staff numbers with the majority employing less than 50 people. Over $70 \%$ of the companies were yet to use cloudbased data storage indicating low adoption of new technology in line with the findings of Marasini et al. (2008) regarding IT adoption in SMEs. Results of the complexity self-assessment show that 54\% of respondents identified their production system as "somewhat complex", 27\% "not very complex" with $19 \%$ reporting "very complex" production processes.

Table 3 Characteristics of Survey Respondents

\begin{tabular}{|l|l|l|l|l|l|l|l|}
\hline \multicolumn{2}{|l|}{ Time in Business } & \multicolumn{2}{l|}{ Annual Turnover } & \multicolumn{2}{l|}{ Total Employees } & \multicolumn{2}{l|}{ Data Stored on Cloud } \\
\hline$<1$ year & $1 \%$ & $<1$ & $32 \%$ & $<10$ & $26 \%$ & None & $71 \%$ \\
\hline $1-5$ years & $4 \%$ & $1-5$ million & $38 \%$ & $11-50$ & $48 \%$ & Some & $19 \%$ \\
\hline 6-10 years & $6 \%$ & $6-10$ million & $12 \%$ & $51-100$ & $13 \%$ & About Half & $5 \%$ \\
\hline$>10$ years & $83 \%$ & $11-50$ million & $14 \%$ & $101-150$ & $6 \%$ & Most & $4 \%$ \\
\hline & & $>50$ million & $4 \%$ & $151-200$ & $2 \%$ & All & $1 \%$ \\
\hline No response & $6 \%$ & No response & $2 \%$ & $>200$ & $5 \%$ & & \\
\hline
\end{tabular}

Figure 1 presents the breakdown of survey responses to the questions on complexity determinants. For the three variability questions (batch size, demand and production), the offered response options of high, medium and low are taken as a proxy for "very", "somewhat", or "not very" complex. The SKU related questions were open-ended to allow respondents enter their approximated values. The intention at the outset of the survey was that responses would be categorized into three groupings 


\section{Byrne, Liston, and Byrne}

corresponding to the three complexity levels and then included in an overall complexity calculation. However on review of the survey responses (Table 3), it became apparent that a very large range of values existed (i.e. 1 to 300,000 for total SKUs and 1 to 15,000 for active SKUs) and that the relative impact of the number of SKUs on complexity was dependent on many factors. These factors include the distinction between individual products (and the consequent implications for machine changeover and processing times), the relative number and complexity of products across companies (i.e. a greater number of simple products versus fewer but more complicated products) and the Pareto breakdown of sales of active SKUs.

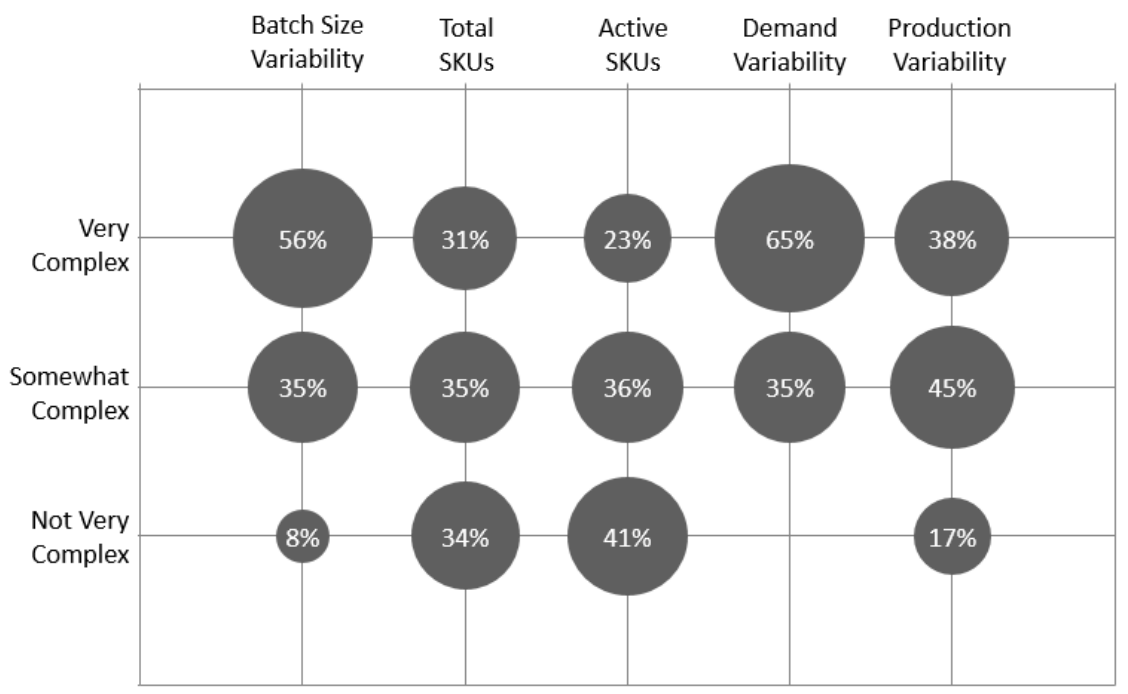

Figure 1 Survey results for selected complexity determinants

For these reasons, the SKU results have been omitted from the calculated average complexity level as presented in Table 4. This table shows that when based on calculated complexity relating to individual responses to the three variability questions (batch size, demand and production) a far greater number of respondent SMEs (174/328 - 53\%) can be categorized as "very complex" versus $19 \%$ of SMEs that based on self-assessment would define themselves as "very complex".

Table 4: Responses received to $S K U$ related questions

\begin{tabular}{|c|c|c|c|c|c|c|}
\hline Complexity Level & Total SKUs & No. & $\%$ & Active SKUs & No. & $\%$ \\
\hline \multirow{3}{*}{ Very } & 300,000 & 1 & \multirow{3}{*}{$31 \%$} & 15,000 & 1 & \multirow{3}{*}{$22 \%$} \\
\hline & $10,000-20,000$ & 4 & & $6,000-8,000$ & 3 & \\
\hline & $1,000-9,000$ & 21 & & $1,000-5,000$ & 14 & \\
\hline \multirow{2}{*}{ Somewhat } & $300-900$ & 11 & \multirow{2}{*}{$35 \%$} & $200-500$ & 19 & \multirow{2}{*}{$38 \%$} \\
\hline & $25-273$ & 19 & & $25-150$ & 12 & \\
\hline Not Very & $0-20$ & 29 & $34 \%$ & $0-20$ & 33 & $40 \%$ \\
\hline
\end{tabular}

Across all respondents, it was found that the majority (approximately 60\%) of companies collect data in some relatively balanced combination of manual and automated processes. Interestingly, when respondents were grouped by their self-assessed levels of complexity (Figure 2), this position was far less pronounced in the case of companies perceived to be "very complex" than was the case in the other two cases (40\% versus circa 65\%). A greater percentage of these companies had mostly automated data collection (30\% versus circa $15 \%$ ) as would possibly be expected in complex environments. However, those with mostly manual data collection also accounted for $30 \%$ of the "very complex" cohort. 
Table 5: Calculated average complexity versus perceived complexity

\begin{tabular}{|l|c|c|c|c|c|c|}
\hline $\begin{array}{l}\text { Complexity } \\
\text { Level }\end{array}$ & $\begin{array}{c}\text { Batch Size } \\
\text { Variability }\end{array}$ & $\begin{array}{c}\text { Production } \\
\text { Variability }\end{array}$ & $\begin{array}{c}\text { Demand } \\
\text { Variability }\end{array}$ & $\begin{array}{c}\text { Total } \\
\text { Responses }\end{array}$ & $\begin{array}{c}\text { Calculated } \\
\text { Complexity }\end{array}$ & $\begin{array}{c}\text { Self-Assessed } \\
\text { Complexity }\end{array}$ \\
\hline Very & 62 & 42 & 70 & 174 & $\mathbf{5 3 \%}$ & $\mathbf{1 9 \%}$ \\
\hline Somewhat & 39 & 49 & 38 & 126 & $\mathbf{3 8 \%}$ & $\mathbf{5 4 \%}$ \\
\hline Not Very & 9 & 19 & 0 & 28 & $\mathbf{9 \%}$ & $\mathbf{2 7 \%}$ \\
\hline $\begin{array}{l}\text { Total } \\
\text { Responses }\end{array}$ & 110 & 110 & 108 & 328 & \multicolumn{2}{|l}{} \\
\cline { 1 - 1 }
\end{tabular}

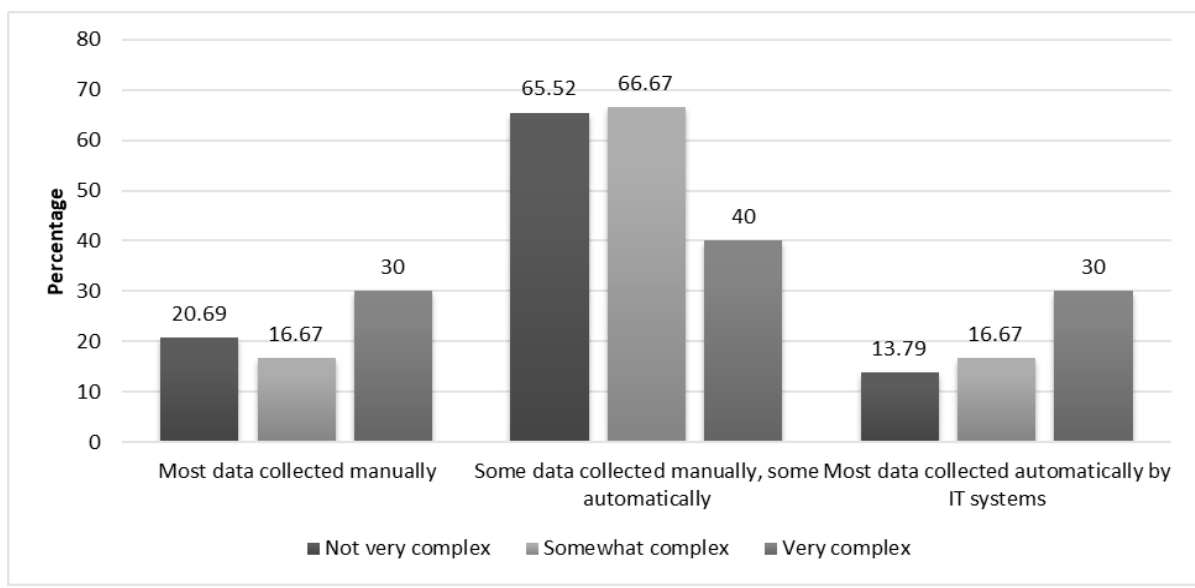

Figure 2 Data Collection Practices presented against Self-Assessed System Complexity

Of those surveyed only $21.4 \%$ of respondents were aware of DES. Figure 3 illustrates that there is a correlation between perceived complexity and awareness of DES with $35 \%$ in the "very complex" category being aware of DES as opposed to $10 \%$ and $22 \%$ of the "not very complex" and "somewhat complex" categories respectively.

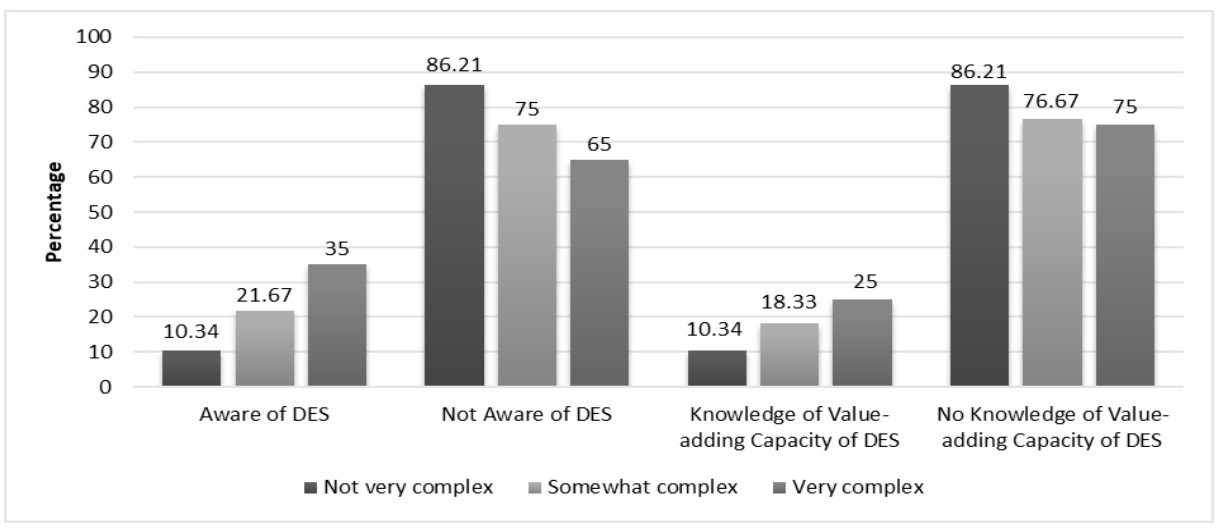

Figure 3 Self-assessed 'awareness of DES' and 'knowledge of value adding capacity of DES'

This correlation could be due to stakeholder motivation to find appropriate analytical methods or due to being more likely targets for vendors of such services. What speaks to the applicability of DES is that a similar trend holds true for respondent ability to identify where DES would add value to their business. As shown, the greater the complexity the more likely that the benefit of DES could be seen. As a percentage of those that were aware of DES in the first place, $70 \%$ of those in very complex environments could see how it would add value to their business. 


\section{DISCUSSION AND CONCLUSIONS}

When questioned directly, a large majority of SMEs identify their business operations as medium or high complexity systems. This in itself would suggest that DES is an appropriate analytical method to apply in these settings as it is particularly suited to modelling complex systems (Robinson, 2005). However, when queried about specific aspects of their business, SMEs show higher levels of complexity with over half of respondents falling into the highest variability category. It is interesting to note that the SMEs surveyed tend to consistently underestimate their overall system complexity when compared to their own assessment of the systems individual components. As evidence of this, the assessment of the combined individual system components places 53\% of the SMEs studied in the highest complexity category versus the self-assessment of only $19 \%$. On the opposite side, the study found that the lowest category (Not Very), only 9\% were calculated as being in this category when based on the systems individual components versus $27 \%$ when based on the respondents selfassessments. This aligns with Park and Okudan Kremer (2015) where they note that it is "very hard for general manufacturing companies to practically identify their current complexity levels at which they operate". Given that SMEs may not fully understand their complexity levels and thus underestimate the usefulness of DES to them, there is an even greater need to ensure DES platforms are presented in an SME usable format and that awareness and training is raised and provided by the SME community. The need for an appropriate pitch is echoed by Löfving et al. (2014) where they suggest that most manufacturing strategy frameworks are prescriptive and developed for larger companies while SMEs require more descriptive frameworks.

Variability is a key driver of uncertainty and one that is difficult to capture in the static spreadsheet analyses that are typically found across manufacturing SMEs. In DES, sources of variability (i.e. stochastic and dynamic system parameters) can be represented and the cumulative effect of these factors can be predicted. From an analysis of the results it is also clear that the levels of complexity experienced by the SMEs studied remain high in both the internal and external categories (Gabriel, (2008); Efthymiou et al., (2012); Blome et al., (2014)). Therefore, the appropriateness of DES to manufacturing SMEs may be even greater as the systems concerned are more complex than the stakeholders perceive. Furthermore, the highest level of variability was reported for customer demand. As an external complexity determinant, this is largely outside the control of the SME but may have an influence on internal complexity, as can be seen in the results for batch size variability. This relationship highlights the potential importance for companies to understand and predict how changes to in-house processes can influence overall performance. Indeed other authors also allude to this being the case (e.g. see Bozarth et al. (2009)) and future work would include performing a deeper analysis to determine the link between external and internal complexity through comparing individual cases. Based on these findings it would suggest that the systems under review are 'sufficiently complex' to warrant the use of DES for particular scenarios.

\section{REFERENCES}

Aelker J, Bauernhansl T and Ehm H (2013) Managing Complexity in Supply Chains: A Discussion of Current Approaches on the Example of the Semiconductor Industry. Procedia CIRP 7. Elsevier B.V.: 79-84.

Anderson P (1999) Perspective: Complexity Theory and Organization Science. Organization Science 10(3).

Beer S (1959) Cybernetics and Management. Wiley.

Blome C, Schoenherr T and Eckstein D (2014) The impact of knowledge transfer and complexity on supply chain flexibility: A knowledge-based view. International Journal of Production Economics 147(PART B). Elsevier: 307-316. DOI: 10.1016/j.ijpe.2013.02.028.

Bozarth CC, Warsing DP, Flynn BB, et al. (2009) The impact of supply chain complexity on manufacturing plant performance. Journal of Operations Management 27(1): 78-93.

Calinescu a, Efstathiou J, Schirn J, et al. (1998) Applying and assessing two methods for measuring complexity in manufacturing. Journal of the Operational Research Society 49(7): 723-733. DOI: $10.1057 /$ palgrave.jors.2600554.

Chance F, Robinson J and Fowler J (1996) Supporting manufacturing with simulation: model design, 
development, and deployment. Proceedings of the 1996 Winter Simulation Conference. DOI: 10.1109/WSC.1996.873268.

Choi TY, Dooley KJ and Rungtusanatham M (2001) Supply networks and complex adaptive systems: control versus emergence. Journal of Operations Management 19(3): 351-366.

Cicirelli F, Furfaro A and Nigro L (2011) Modelling and simulation of complex manufacturing systems using statechart-based actors. Simulation Modelling Practice and Theory 19(2). Elsevier B.V.: 685-703.

De Toni AF De, Nardini A, Nonino F, et al. (2001) Complexity measures in manufacturing systems. Bourgine P., Kepes F. SM (ed.) Proceedings of the European Conference on Complex Systems Towards a science of complex systems: $1-20$.

Deshmukh a. V, Talavage JJ and Barash MM (1992) Characteristics of part mix complexity measure for manufacturing systems. Proceedings of the 1992 IEEE International Conference on Systems, Man, and Cybernetics: 1384-1389. DOI: 10.1109/ICSMC.1992.271590.

Deshmukh A V, Talavage JJ and Barash MM (1998) Complexity in Manufacturing Systems Part 1 : Analysis of Static Complexity. IIE Transactions 30(7): 645-655. DOI: 10.1023/A:1007542328011.

Efthymiou K, Pagoropoulos a., Papakostas N, et al. (2012) Manufacturing systems complexity review: Challenges and outlook. Procedia CIRP 3(1): 644-649. DOI: 10.1016/j.procir.2012.07.110.

ElMaraghy W, ElMaraghy H, Tomiyama T, et al. (2012a) Complexity in engineering design and manufacturing. CIRP Annals - Manufacturing Technology 61(2). CIRP: 793-814. DOI: 10.1016/j.cirp.2012.05.001.

ElMaraghy W, ElMaraghy H, Tomiyama T, et al. (2012b) Complexity in engineering design and manufacturing. CIRP Annals - Manufacturing Technology 61(2). CIRP: 793-814. DOI: 10.1016/j.cirp.2012.05.001.

Fowler JW and Rose O (2004) Grand Challenges in Modeling and Simulation of Complex Manufacturing Systems. In: Simulation, 1 September 2004, pp. 469-476. DOI: $10.1177 / 0037549704044324$.

Fredendall LD and Gabriel TJ (2003) Manufacturing Complexity: A Quantitative Measure. In: Proceedings of POMS 14th Annual Conference, Savannah, Georgia, 2003.

Frizelle G and Suhov Y (2008) The measurement of complexity in production and other commercial systems. In: Proceedings of the Royal Society A: Mathematical, Physical and Engineering Sciences, 2008, pp. 2649-2668. DOI: 10.1098/rspa.2007.0275.

Frizelle G and Woodcock E (1995) Measuring complexity as an aid to developing operational strategy. International Journal of Operations \& Production Management 15(5): 26-39. DOI: $10.1108 / 01443579510083640$.

Gabriel (2008) Measuring the manufacturing complexity created by system design. In: Proceedings of the 38th Annual Meeting of the Southeast Region of the Decision Sciences Institute (SEDSI), Orlando, Florida, USA, 2008.

Garzon AL, Arokiam A and Greig N (2012) Complexity of scheduling in SMEs specialising in High Variety Low value marketing. In: KTP Associates Conference, University of Brighton, 2012.

Götzfried M (2013) Managing Complexity Induced by Product Variety in Manufacturing Companies. Complexity Evaluation and Integration in Decision-Making. University of St. Gallen.

Ingemansson A, Bolmsjö GS and Harlin U (2002) A Survey of the Use of the Discrete-Event Simulation in Manufacturing Industry. In: Proceedings of the 10th International Manufacturing Conference in China (IMCC2002), 2002.

Isik F (2010) An Entropy-Based Approach for Measuring Complexity in Supply Chains. International Journal of Production Research 12: 3681-3696.

Johansson M, Jahansson B, Leong S, et al. (2008) A real world pilot implementation of the core manufacturing simulation data model. In: Proceedings of the Simulation Interoperability Standards Organization (SISO) Spring 2008 SIW Workshop, 2008.

Lang M, Deflorin P, Dietl H, et al. (2014) The Impact of Complexity on Knowledge Transfer in Manufacturing Networks. Production and Operations Management 23(11): 1886-1898. DOI: 10.1111/poms.12193. 
Lewin AY (1999) Application of Complexity Theory to Organization Science. Organization Science 10(3).

Löfving M, Säfsten K and Winroth M (2014) Manufacturing strategy frameworks suitable for SMEs. Journal of Manufacturing Technology Management 25(1): 7-26. DOI: 10.1108/JMTM-08-20120081.

Meyer MH and Curley KF (1995) The impact of knowledge and technology complexity on information systems development. Expert Systems with Applications 8(1): 111-134. DOI: 10.1016/0957-4174(94)E0003-D.

Miller JH and Page SE (2017) Complex Adaptive Systems: An Introduction to Computational Models of Social Life. Princeton University Press.

Modrak V and Marton D (2012) Modelling and complexity assessment of assembly supply chain systems. Procedia Engineering 48: 428-435. DOI: 10.1016/j.proeng.2012.09.536.

Modrak V and Marton D (2013) Structural complexity of assembly supply chains: A theoretical framework. Procedia CIRP 7. Elsevier B.V.: 43-48. DOI: 10.1016/j.procir.2013.05.008.

Mönch L (2007) Simulation-based benchmarking of production control schemes for complex manufacturing systems. Control Engineering Practice 15(11): 1381-1393. DOI: 10.1016/j.conengprac.2006.05.010.

Park K and Okudan Kremer GE (2015) Assessment of static complexity in design and manufacturing of A product family and its impact on manufacturing performance. International Journal of Production Economics. Elsevier. DOI: 10.1016/j.ijpe.2015.07.036.

Pickering A (2004) The science of the unknowable: Stafford Beer's cybernetic informatics. Kybernetes 33(3/4): 499-521.

Pradhan S and Damodaran P (2009) Performance characterization of complex manufacturing systems with general distributions and job failures. European Journal of Operational Research 197(2). Elsevier B.V.: 588-598. DOI: 10.1016/j.ejor.2008.07.013.

Robinson S (2005) Discrete-event simulation: from the pioneers to the present, what next? Journal of the Operational Research Society 56(6): 619-629. DOI: 10.1057/palgrave.jors.2601864.

Serdarasan S (2013) A review of supply chain complexity drivers. Computers and Industrial Engineering 66(3). Elsevier Ltd: 533-540. DOI: 10.1016/j.cie.2012.12.008.

Simon HA (1962) The Architecture of Complexity. Proceedings of the American Philosophical Society 106(6): 467-482.

Zhang Z (2011) Modeling complexity of cellular manufacturing systems. Applied Mathematical Modelling 35(9). Elsevier Inc.: 4189-4195. DOI: 10.1016/j.apm.2011.02.044.

\section{AUTHOR BIOGRAPHIES}

DR JAMES BYRNE is an Assistant Professor in Operations and Analytics in DCU Business School, Ireland. In the past, he has worked with SAP Research (UK) as a Senior Research Scientist in the Technology Infrastructure domain, the Enterprise Research Centre in the University of Limerick. He has been co-PI and has lead work packages on a number large EU funded collaborative research projects including OPTIMIS (FP7), CACTOS (FP7) and RECAP (H2020).

DR PAUL LISTON is a Lecturer in Data Analytics at Athlone Institute of Technology. Paul previously held the position of Research Fellow at Dublin City University and undertook the roles of Global Commodity Manager and Supply Chain Consultant with Dell Computers. Prior to this, Paul worked with the Irish Centre for Manufacturing Research in the area of process improvement with companies including Analog Devices, Bombardier, Boston Scientific, Seagate, HP, Intel and Pfizer.

PROF PJ BYRNE is a Professor of Operations Management in Dublin City University Business School. Prior to joining DCU he worked in the University of Limerick from $2004-2007$. He has an extensive track record in industrial based research and was a founding member of the Modelling and Simulation Research Group in DCUBS. He has been a PI for a number of international research projects in the areas of DES and its application in manufacturing, services and healthcare. 